\title{
Myelodysplastic Syndrome with Ring Sideroblasts and Multilineage Dysplasia
}

National Cancer Institute

\section{Source}

National Cancer Institute. Myelodysplastic Syndrome with Ring Sideroblasts and

Multilineage Dysplasia. NCI Thesaurus. Code C27726.

A myelodysplastic syndrome characterized by bi-cytopenia or pancytopenia, dysplastic changes in $10 \%$ or more of the cells in two or more of the myeloid cell lines, and $15 \%$ or more ring sideroblasts in the bone marrow. (WHO, 2001) 